Annales Geophysicae, 23, 2081-2094, 2005

SRef-ID: 1432-0576/ag/2005-23-2081

(C) European Geosciences Union 2005

\title{
The effect of $E$-region wave heating on electrodynamical structures
}

\author{
J.-M. A. Noël ${ }^{1}$, J.-P. St.-Maurice ${ }^{2}$, and P.-L. Blelly ${ }^{3}$ \\ ${ }^{1}$ Department of Physics, Royal Military College of Canada, Kingston, Ontario, Canada \\ ${ }^{2}$ Department of Physics and Engineering Physics, The University of Saskatchewan, Saskatoon, Saskatchewan, Canada \\ ${ }^{3}$ Laboratoire de Physique et Chimie de l'Environnement, Orléans, France
}

Received: 16 September 2004 - Revised: 22 March 2005 - Accepted: 20 June 2005 - Published: 15 September 2005

\begin{abstract}
We show that heating by large amplitude $E$-region plasma waves at high latitudes can at times substantially enhance the electro-dynamical response of the ionosphere. This is made manifest through an increase in parallel current densities and parallel electric fields generated at the edge of arcs in the $E$ and lower $F$-region of the ionosphere, in response to sharp cutoffs in precipitation with an otherwise uniform differential energy flux. The enhancement is rooted in a reduction in electron recombination that occurs in response to higher electron temperatures triggered by the generation of strong electric fields near the edge of the arc. The reduced recombination rate, in turn, leads to enhanced conductivity gradients near the edge of the arc, which, in turn, drives more intense parallel currents and stronger local electric fields.
\end{abstract}

Keywords. Ionosphere (Electric fields and curents; Plasma temperature and density) - Space plasma physics (Numerical simulation studies)

\section{Introduction}

St.-Maurice et al. (1996) have argued that a host of unusual phenomena sometimes seen near auroral arcs can be explained in terms of electro-dynamical phenomena in the presence of unusual precipitation patterns. The unusual observations include very large shears in the horizontal plasma drifts near certain arcs, large magnetic perturbations detected on board satellites or rockets, as well as the observation by incoherent scatter radars of large amplitude ion-acoustic waves along the geomagnetic field line. Noël et al. (2000) have therefore studied in some detail the electrodynamics of twodimensional auroral arcs which, instead of being associated with inverted $\mathrm{V}$ precipitation patterns, would be triggered by a flat precipitation pattern coupled with a sharp horizontal/latitudinal cutoff $(200 \mathrm{~m})$. They showed that the introduction of this kind of precipitation pattern did not lead to

Correspondence to: J.-M. A. Noël

(jean-marc.noel@rmc.ca) the usual short-circuiting of the perpendicular electric field and weak parallel current densities associated with "normal" inverted-V arcs. Instead, they obtained thermal parallel current densities that became extremely large at the edges of the arc while the perpendicular electric field was perturbed only near the edges, with very little change short-circuiting effects near the center of the arc. In addition, the perpendicular electric field became larger just outside the arc and weaker just inside the arc. To be more precise, Noël et al. (2000) showed that, consistent with back-of-the-envelope calculations for such situations, parallel current densities of several $100 \mu \mathrm{A} / \mathrm{m}^{2}$ could be carried by thermal electrons, provided that a sufficiently large DC electric field or a sufficiently sharp drop in precipitation flux was used. With 200-m horizontal cut-off scales in precipitation, ambient perpendicular electric fields of the order of $50 \mathrm{mV} / \mathrm{m}$ or more were required.

The Noël et al. (2000) calculations included a state-ofthe-art description of the chemistry and of the transport and electron cascading processes along the geomagnetic field lines. A coupling of the various field lines was introduced by imposing the condition that the currents in the system be divergence-free. However, one process that was not included in the original calculations was the repeated observed heating of electrons at $E$-region heights in the presence of strong electric fields. In this paper we have now included this heating. We are able to show that the additional electron heating has a surprisingly strong effect on the results, in that it actually increases further the parallel electric fields and currents at the edges of the kinds of arcs that trigger strong parallel current responses at their edges.

Since our work introduces the contribution of electron heating to the electro-dynamical ionospheric feedback, for the first time, we feel that a brief overview of the subject should be in order.

Ever since the first clear observations reported by Schlegel and St.-Maurice (1981) and Wickwar et al. (1981) it has become obvious that the $E$-region electron temperature is enhanced in the presence of large amplitude plasma irregularities at high latitudes. Two main mechanisms have been 
invoked to connect the irregularities to electron heating. The first has been associated with anomalous perpendicular diffusion, in effect, enhanced broadband perpendicular fields (Robinson, 1986; St.-Maurice, 1987). The second has invoked wave parallel fields, since it is well known that the irregularities can be a few degrees off perpendicularity to the magnetic field under strong DC electric field conditions (St.Maurice and Laher, 1985; St.-Maurice, 1990b; Milikh and Dimant, 2002; Dimant and Milikh, 2003; Milikh and Dimant, 2003).

In our work it is important to have a quantitative description of the electron heating effects that is both easy to program and that has been tested against observations. Two such descriptions currently exist, one for each of the proposed wave mechanisms. Both descriptions have been claimed to work well. The one from Robinson (1986), which relies on anomalous diffusion, is particularly simple to use and it has been tested repeatedly and shown to work well. However, another expression has recently been proposed by Dimant and Milikh (2003), using the parallel wave heating mechanism. These latter authors have also claimed that their formula works, though on the face of it, it certainly looks quite different, as we show below. Particularly in the large electric field regime that interests us here, a regime that has not been tested as thoroughly, there may be some differences between the two prescriptions.

While the Robinson (1986) formula has been tested more thoroughly and is simpler to use, there are physical reasons why one would come to favor the more complicated and less tested Dimant and Milikh (2003) expressions. For instance, the Robinson (1986) saturation mechanism involves a diffusive process which is at right angles to the one required to saturate the waves (St.-Maurice, 1990a). Another troubling point out is that St.-Maurice and Hamza (2001) have pointed in their work on intermittency that the electric field inside the irregularities is actually smaller than the ambient field: the presence of the structures should, after all, act to short out electric fields within the structures themselves, not enhance them. This implies that the total heating rate should go down, not up, in the presence of perfectly field-aligned structures, since the field in such structures is systematically less than the ambient field. This point is often overlooked in linear theories, so that when the linear results are used in quasi-linear expressions they lead to erroneous results.

In the end, we have simply chosen to study the effect of electron heating using the two separate prescriptions. The Robinson (1986) prescription has the virtue of having been repeatedly tested and shown to do well. However, the less tested Dimant and Milikh (2003) prescription has a different physics that may well be working better in the end. Therefore, with claims from both sides that the formulae agree well with observations, we can provide with our work a test of whether or not this is the case, and whether or not apparently small differences can have important consequences.

Given the background described above, we will, in the present paper, focus once again on the modelling of elongated auroral structures created by uniform particle precipi- tation with sharp cut-offs in their latitudinal pattern. We will once again add the presence of large ambient electric fields, so as to trigger large parallel current densities on the edge of the structures. The goal will simply be to quantify and understand the effects of electron heating by Farley-Buneman waves, first on the energetics, and then on the composition and the electrodynamics.

The rest of our paper unfolds as follows: in Sect. 2 we describe our electrodynamic model, as well as the modifications that were made to the transport model, so as to take into account the wave-induced electron heating. In Sect. 3 we present results from our model for the cases without anomalous electron wave heating, with wave heating using Robinson's expression and finally, with wave heating using Dimant and Milikh's expression. We end with a summary and conclusion in Sect. 4.

\section{The Model}

\subsection{Basic description}

A complete description of the basic model used in our electrodynamical calculation, as well as the numerical technique, can be found in Noël et al. (2000). We only briefly review the procedure here.

The model is two-dimensional in $x$ (north-south) and $z$ (magnetic field) directions. The east-west, or $y$-derivatives, are neglected under the assumption of an elongated eastwest precipitation pattern and arc. Aside from this, the model is made of two distinct parts. The first part consists of the comprehensive time-dependent transport TRANSCAR model, which has been used as a basis for many studies over the years. The second part of the model is an electrodynamical model which we call ELECTRO. Our main focus here will be on the equations that we modified in order to add an electron $E$-region wave heating term.

A complete description of TRANSCAR is provided in Blelly et al. (1996). In short, TRANSCAR is a onedimensional program that models the terrestrial ionosphere in the altitude range $100-3000 \mathrm{~km}$, along the geomagnetic field. It itself consists of two parts. The first part of TRANSCAR is a fluid description of the ionosphere and is based on the 8-moment transport formulation (Blelly and Schunk, 1993). The model includes six thermal ion species $\left(\mathrm{O}^{+}\right.$, $\mathrm{H}^{+}, \mathrm{N}^{+}, \mathrm{NO}^{+}, \mathrm{N}_{2}^{+}$and $\mathrm{O}_{2}^{+}$), as well as thermal electrons. It computes the temporal evolution of the concentrations, the field-aligned velocities, the average temperatures and the field-aligned heat flows for each species as well as for the thermal electrons. The second part of TRANSCAR is a kinetic transport model of energetic electrons which accounts for the precipitation of electrons originating from the magnetosphere and for photoelectrons resulting from the influence of the solar EUV flux on the neutral atmosphere (Lilensten et al., 1989; Lummerzheim and Lilensten, 1994; Blelly et al., 1996; Blelly et al., 2005; Lilensten and Blelly, 2002). The 
kinetic model provides the ion production rates and electron heating source for the fluid model.

In Fig. 1, we present the precipitating electron spectral flux that was used in simulations for both the present study and the one published by Noël et al. (2000). The total particle flux is $2.3 \times 10^{13} \mathrm{~m}^{-2} \mathrm{~s}^{-1}$ and corresponds to an energy flux of $6 \mathrm{~mW} \mathrm{~m}^{-2}$ into the ionosphere. This spectral flux was chosen because the energy of the precipitating electrons was high enough to penetrate deep into the $E$-region and to ionize it. The shape of the spectral flux is in agreement with those that can be found in a number of references (e.g., Rees, 1989; Blelly et al., 1996).

The electrodynamical part of the model, ELECTRO, is based on an approach first suggested by St.-Maurice et al. (1996) and Noël et al. (2000). It assumes that the current density is divergence-free, which is not a problem for the time and spatial scales of interest,

$\nabla \cdot \boldsymbol{J}=0$.

This equation means that for the temporal and spatial scales of interest, the perturbed electric fields and resulting currents are adjusting instantaneously to any temporal change in conductivity or precipitation input.

An important point about the procedure used to solve the problem at hand is that we do not make any assumption about the magnitude of the "perturbed" electric fields. We simply separate the current density $\boldsymbol{J}$ into three parts and carry on with the calculations. The first part is made up of a source term due to precipitation, $\boldsymbol{J}_{s}^{p}$, i.e. it describes the currents carried by precipitating fluxes of energetic electrons. The behavior of that current source is modelled through the kinetic part of TRANSCAR mentioned earlier. The second source term is related to the presence, prior to the introduction of precipitation, of a uniform background electric field to which computed conductivity gradients will be added. Finally, we have the most important contribution in our work by far, namely, a "thermal response term", $\boldsymbol{J}_{t h}$, which depends on the perturbed electric field or potential, as well on the conductivities.

The last two current density terms are described with the use of a simple Ohm's law, namely, from the equation

$\boldsymbol{J}=\boldsymbol{\sigma} \cdot \boldsymbol{E}$,

where $\sigma$ is the classical conductivity tensor, and $\boldsymbol{E}$ is the electric field. The conductivity tensor is, in turn, given by

$\boldsymbol{\sigma}=\left(\begin{array}{ccc}\sigma_{P} & \sigma_{H} & 0 \\ -\sigma_{H} & \sigma_{P} & 0 \\ 0 & 0 & \sigma_{\|}\end{array}\right)$,

where $\sigma_{P}, \sigma_{H}$ and $\sigma_{\|}$are the usual Pedersen, Hall and parallel conductivities, respectively.

As stated above, we have divided the electric field into two parts, namely, $\boldsymbol{E}_{=} \boldsymbol{E}_{0}-\nabla \phi$, where $\boldsymbol{E}_{0}$ is a constant background electric field and $\phi$ is the electrostatic potential of the

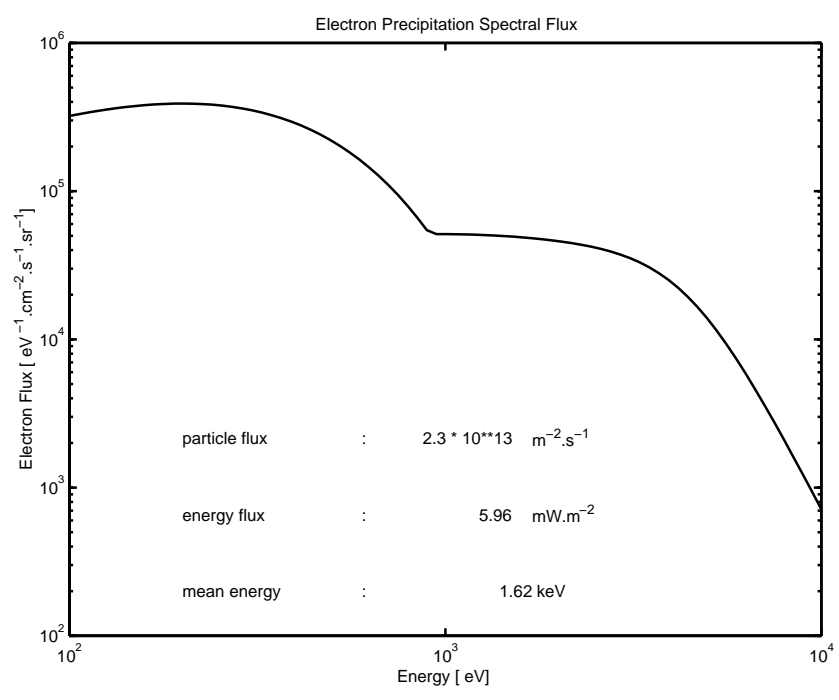

Fig. 1. Spectral flux of the precipitating electrons.

disturbed field caused by the creation of the auroral arc introduced by the electron precipitation at time $t=0$. As a result Eq. (1) becomes

$\nabla \cdot \boldsymbol{J}=\nabla \cdot\left(\boldsymbol{\sigma} \cdot \boldsymbol{E}_{0}\right)+\nabla \cdot \boldsymbol{J}_{s}^{p}-\nabla \cdot(\boldsymbol{\sigma} \cdot \nabla \phi)=0$.

Since we consider $\boldsymbol{E}_{0}$ to be constant and uniform and since $\sigma$ is computed using TRANSCAR's output, the first term on the right-hand-side of Eq. (4) can be viewed as another source term by ELECTRO. Given that the ambient electric field $\boldsymbol{E}_{0}$ is assumed to be perpendicular to the geomagnetic field and that we assume the $y$-direction to be derivative-free, we rewrite this first term as

$\nabla \cdot\left(\boldsymbol{\sigma} \cdot \boldsymbol{E}_{0}\right)=E_{x}^{0} \frac{\partial \sigma_{P}(x, z)}{\partial x}+E_{y}^{0} \frac{\partial \sigma_{H}(x, z)}{\partial x}$,

where the terms $\partial \sigma_{P, H} / \partial x$ are the horizontal north-south gradients in the Pedersen/Hall conductivities that are created at the edge of the precipitating region. For simplicity we limit ourselves for now to the case where $E_{y}^{0}$ is negligibly small.

The second term on the right-hand-side of Eq. (4) is a source due to the current carried by precipitating electrons. These high energy electrons play a very important role in our calculations because they greatly enhance the Pedersen conductivities in the precipitating region. In addition, with the imposition of sharp latitudinal cutoff in precipitation, they introduce sharp Pedersen conductivity gradients. In turn, these conductivity gradients drive a strong response in both the first and last terms of Eq. (4). Interestingly enough, however, the precipitating currents themselves play no significant direct role in Eq. (4). This is easy to see if we compare the magnitudes of the first and second terms on the right-hand side of Eq. (4), using a $10 \mathrm{mV} / \mathrm{m}$ field combined with a $100-\mathrm{m}$ scale in the precipitation cutoff. In that case the first term in Eq. (4) is of the order of $10^{-4} \mathrm{~A} / \mathrm{m}^{3}$. We can contrast this with the second term, where our currents are $3 \mu \mathrm{A} / \mathrm{m}^{2}$, if we assume, very optimistically, that the precipitating currents diverge strongly over a $1-\mathrm{km}$ scale in the vertical (easily 


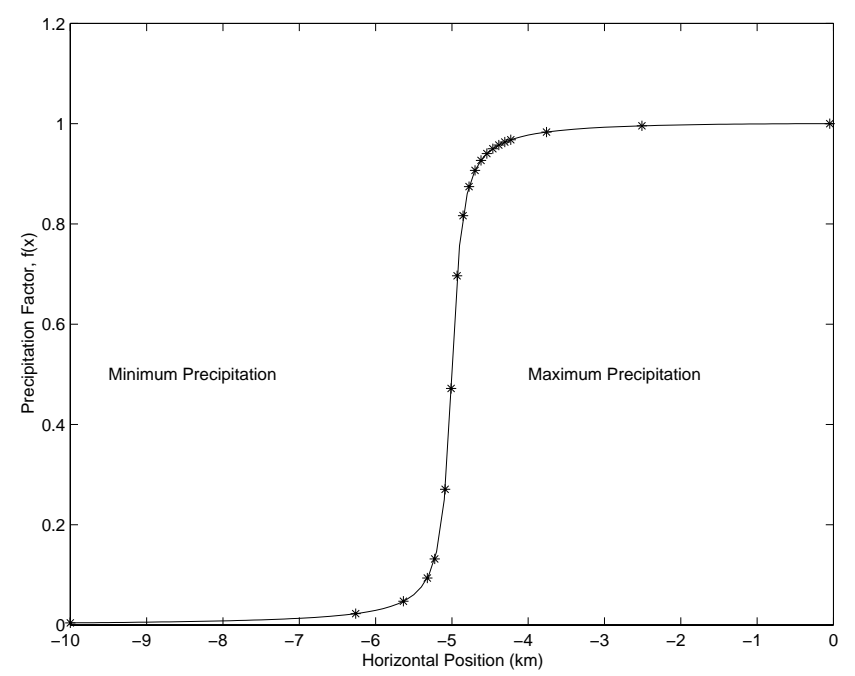

Fig. 2. Figure showing the field lines used in the computation.

one order of magnitude too small a scale). Even with this optimistic number, however, we only obtain $10^{-9} \mathrm{~A} / \mathrm{m}^{3}$, which is totally negligible by comparison to the first term. We verified, through numerical runs made with and without it, that the precipitating currents indeed played no role through the presence of the second term on the right-hand side of Eq. (4). Therefore, in order to speed up the calculations, we have, after these tests, simply discarded the term in question.

After neglecting the divergence in precipitating currents, and with the help of Eq. (5) we have rewritten Eq. (4) in the form

$$
\begin{aligned}
& \frac{\partial}{\partial x}\left(\sigma_{P}(x, z) \frac{\partial \phi(x, z)}{\partial x}\right)+\frac{\partial}{\partial z}\left(\sigma_{\|}(x, z) \frac{\partial \phi(x, z)}{\partial z}\right) \\
& \quad \approx E_{x}^{0} \frac{\partial \sigma_{P}(x, z)}{\partial x},
\end{aligned}
$$

where $\phi(x, z)$ is the electric potential introduced by the horizontal and "vertical" gradients in conductivities triggered by the precipitation that we introduce at time $t=0$.

For the conductivities, we use standard expressions that may be found in a number of references. These expressions are presented in detail in Noël et al. (2000) and will not be repeated here. The only point to stress is that the conductivities are not only functions of altitude $z$ but also functions of the latitudinal position $x$, through changes in the plasma density that are introduced by the arc. From Eq. (6) we can then see that a structuring of the conductivities has to cause a structuring of the electric potential and, therefore, of the associated electric fields and current densities.

Once the electric fields and current densities as a function of position have been determined using ELECTRO, they are fed into the transport equations (TRANSCAR) to determine the new concentrations, temperatures, field-aligned velocities and field-aligned heat flows. The resulting densities and temperatures are then returned to ELECTRO to compute the changes in the conductivities and the new electric potential via Eq. (6) and the associated electric fields and current densities. The iterations continue in this way as time advances.

Figure 2 describes the function used to model the latitudinal distribution of the electron precipitation in our simulation. The asterisks indicate the field lines simulated by TRANSCAR. Note that we concentrate the field lines in the region of the maximum gradients in the precipitation profile. This is necessary in order to model the sharp precipitation gradients with adequate coverage.

A summary of the physics that we study with our model unfolds as follows: At first, precipitation creates regions of enhanced electron densities that modify the conductivities. Since we have a region of enhanced conductivities there must exist a gradient in the conductivities, concentrated, in this case, near the edge of the structure. This conductivity gradient rearranges the electric potential and associated electric field around the edge of the structure. The modified field, depending on the sign of its perturbation, can locally enhance or decrease the Joule heating rate of ions, as well as electrons. In the latter case the frictional heating rate is effectively increased by close to one order of magnitude in the 100 to $120 \mathrm{~km}$ region, owing to the heating by the $E$-region plasma irregularities discussed in the Introduction. It is the effect of this additional electron heating term that we wish to examine in detail in the present work in light of our 2-D electrodynamical model. Simply put, elevated electron temperatures reduce the recombination rate (e.g., Sheehan and St.Maurice, 2004). This means enhanced densities and therefore higher conductivities in the hotter regions. This, in turn, modifies the conductivity gradients, and through them, the parallel currents and the ambient electric fields. We show below that the feedback can be positive and substantial for the steep precipitation cutoffs under study here, and we describe how the feedback operates, by studying the results of our calculations with and without the electron wave heating term.

\subsection{Modifications brought to the standard TRANSCAR model}

The equations used in the fluid formulation of the transport part of TRANSCAR may be found in Blelly and Schunk (1993) and Blelly et al. (1996). We only discuss here our modifications to the electron energy equation in response to $E$-region wave heating of electrons.

The Joule heating rate contribution from species $s$ normally used by TRANSCAR is given by the standard expression (e.g., Schunk and Nagy, 2000)

$Q_{E}^{s}=\frac{n_{s} e_{s}^{2} v_{s} E_{\perp}^{2}}{m_{s}\left(v_{s}^{2}+\Omega_{s}^{2}\right)}$,

where $E_{\perp}$ is the perpendicular convective electric field amplitude measured from the neutral frame of reference, $B$ is the magnetic field strength, $\Omega_{s}=e_{s} B / m_{s}$ is the gyrofrequency of species $s, e_{s}$ is the electronic charge of species 
$s, v_{s}=\sum_{t} m_{s} v_{s t} /\left(m_{s}+m_{t}\right)$ is the momentum transfer frequency between ionized species $s$ and species $t, m_{s}$ and $m_{t}$ are the masses of species $s$ and $t$, respectively, and $Q_{E}^{s}$ is the heating rate of species $s$ due to the perpendicular electric field.

\subsubsection{Robinson's expression for the electron heating rate}

In TRANSCAR, the classical heating rate for the electrons has been modified to include the electron heating rate by plasma waves. For a first set of calculations, we used the expressions developed by Robinson (1986). According to Robinson's prescription, the wave heating rate is described as follows: for $v_{d}<c_{s}$ we use the classical heating rate for the electrons which reads

$Q_{e}=Q_{\text {classical }}=\frac{n_{e} e^{2} v_{e} E_{\perp}^{2}}{m_{e}\left(v_{e}^{2}+\Omega_{e}^{2}\right)}$.

However, if $v_{d}>c_{s}$, it can easily be shown that Robinson's heating rate is given instead by the expression

$Q_{e}=Q_{\text {classical }}+\frac{\Omega_{i} \Omega_{e} m_{e}}{v_{i}} \frac{\left(v_{d}-c_{s}\right)^{3}}{c_{s}}$,

where $v_{d}$ is the magnitude of the $\boldsymbol{E} \times \boldsymbol{B}$ drift and the electric field, as always, is measured in the neutral frame of reference. Also, $c_{s}=\sqrt{k_{b}\left(T_{i}+T_{e}\right) / m_{i}}$ is the ion-acoustic speed, where $k_{b}$ is the Boltzmann constant, $T_{i}$ and $T_{e}$ are the ion and electron temperatures, respectively, and $m_{i}$ is the average ion mass.

\subsubsection{Dimant and Milikh's heating rate}

More complicated expressions, but also probably more physically correct ones, were recently derived by Dimant and Milikh (2003), to describe the heating of electrons by wave parallel electric fields. Given the complexity of the problem, the authors had to use a heuristic model of the saturated turbulent electric field, but ended up, nevertheless, with expressions that seemed to reproduce the observations well. This particular wave heating model leads to a temperature expression described by

$$
\begin{aligned}
\frac{\Delta T_{e}}{T_{0}} & =\frac{4 \kappa_{i}^{2} v_{i n}}{3 \delta_{e n} v_{e n}} \frac{E_{C}^{2}}{E_{00}^{2}}\left\{\left(\frac{1+\psi_{\perp}}{1+\kappa_{i}^{2}}\right) \frac{\left(E_{C}-E_{T h r}\right)^{3}}{E_{C}^{2} E_{T h r}}\right. \\
& \left.+\psi_{\perp}\left[1+\left(1-\frac{E_{T h r}}{E_{C}}\right)^{2}\right]\right\},
\end{aligned}
$$

where $\delta_{e n} v_{e n}$ is electron energy loss rate, while $\Delta T_{e}=T_{e}-T_{0}$ is the temperature increment and $T_{e}$ is the actual electron temperature while $T_{0}$ is, in effect, the neutral temperature. The amplitude of the DC electric field is given by $E_{C}$ and the dimensionless parameters $\psi_{\perp}$ and $\kappa_{i}$ are defined by

$\psi_{\perp}=\frac{v_{e n} \nu_{i n}}{\Omega_{e} \Omega_{i}}$

and

$\kappa_{i}=\frac{\Omega_{i}}{v_{i n}}$, where $\Omega_{i}$ is the gyrofrequency of the ions and $v_{e n}, v_{i n}$ are the collision frequencies with the neutrals. The threshold electric field, $E_{T h r}$, is given by

$E_{T h r}=\left(1+\psi_{\perp}\right)\left(\frac{1+\kappa_{i}^{2}}{1-\kappa_{i}^{2}}\right)^{1 / 2} E_{0}$,

where

$E_{0}=c_{s} B_{0}=\sqrt{\frac{k_{B}\left(T_{e}+T_{i}\right)}{m_{i}}} B_{0}$,

where $B_{0}$ is the geomagnetic field amplitude and $m_{i}$ is the average ion mass.

Finally,

$E_{00}=\sqrt{\frac{2 k_{B} T_{0}}{m_{i}}} B_{0}$

is the Farley-Buneman threshold electric field in the undisturbed ionosphere.

We notice that $\psi_{\perp}$ depends on $T_{e}$ through the momentum transfer collision frequency $v_{e n}$. If we consider that the neutral atmosphere consists mainly of $\mathrm{N}_{2}, \mathrm{O}_{2}$ and $\mathrm{O}$, we have from Table 4.6 of Schunk and Nagy (2000) the following expressions for the electron-neutral momentum transfer collision frequencies, in $\mathrm{s}^{-1}$ :

$e$ and $\mathrm{N}_{2}$ :

$v\left(e, \mathrm{~N}_{2}\right)=2.33 \times 10^{-11} n\left(\mathrm{~N}_{2}\right)\left(1-1.21 \times 10^{-4} T_{e}\right) T_{e} ;$

$e$ and $\mathrm{O}_{2}$ :

$\mathcal{y}\left(e, \mathrm{O}_{2}\right)=1.82 \times 10^{-10} n\left(\mathrm{O}_{2}\right)\left(1+3.6 \times 10^{-2} \sqrt{T_{e}}\right) \sqrt{T_{e}}$

$e$ and $\mathrm{O}$ :

$v(e, \mathrm{O})=8.9 \times 10^{-11} n(\mathrm{O})\left(1+1.57 \times 10^{-4} T_{e}\right) \sqrt{T_{e}}$.

In these expressions, the densities are in $\mathrm{cm}^{-3}$ and the temperatures in K. Clearly, if $T_{e}$ is structured, then so is $\psi_{\perp}$ and consequently $E_{T h r}$. Furthermore, if $E_{C}$ is horizontally structured, $T_{e}$ has to reflect that structure as well.

\section{Results}

We now present the numerical results from three simulations using TRANSCAR and ELECTRO, five minutes after the onset of electron precipitation into the system. The first simulation describes the results without electron heating due to waves. We use this run as a benchmark against which the other simulations, which contain the new heating rates, will be compared. In all of the runs, the ambient convective electric field was chosen to be $100 \mathrm{mV} / \mathrm{m}$ and oriented exactly along the $x$ direction. We have chosen to model a $10-\mathrm{km}$ wide arc with a 200-m horizontal gradient scale for the precipitation cutoff. 

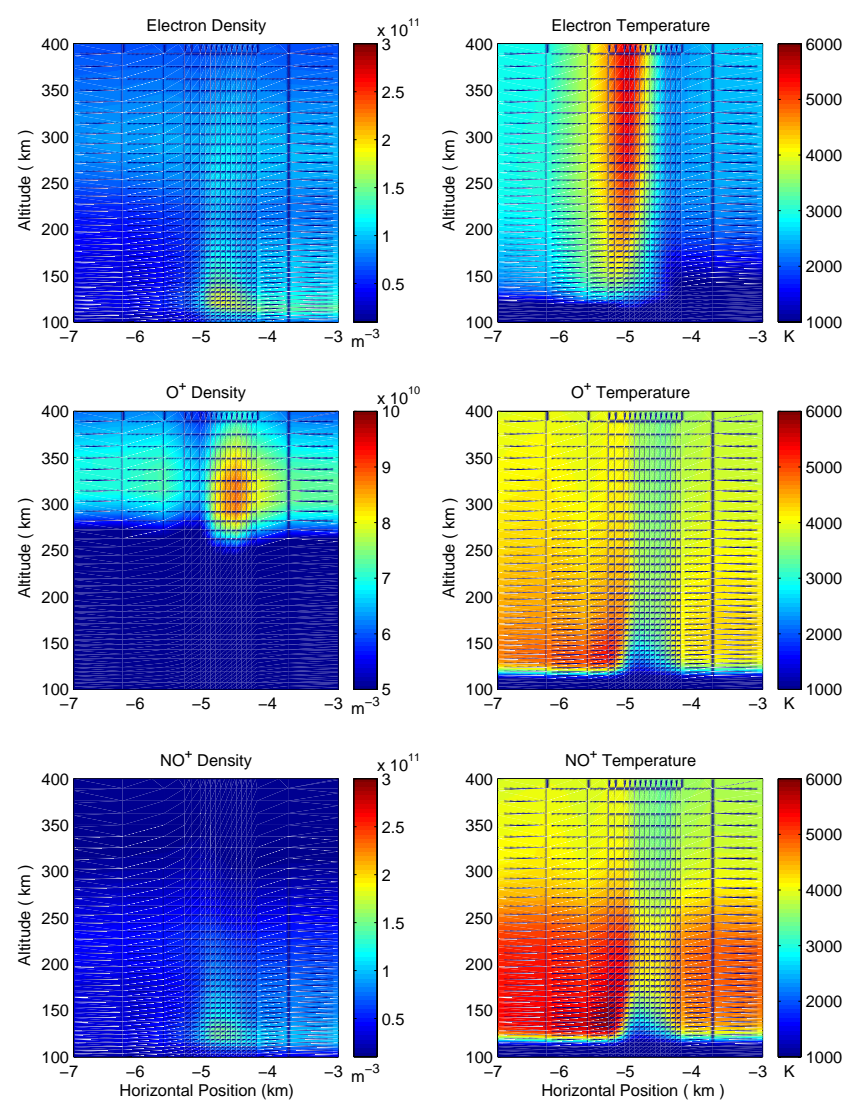

Fig. 3. TRANSCAR output in the absence of $E$-region electron heating by plasma waves and with the precipitation flux given in Fig. 1.

We note that the effects of the $E$-region electron heating on the energetics, composition, electrodynamics and the feedbacks between them, would simply not be taking place without a large magnitude for the electric field, since the heating only becomes considerable once the electric field exceeds $50 \mathrm{mV} / \mathrm{m}$. We have therefore limited our study here to the $100 \mathrm{mV} / \mathrm{m}$ case and to the sharp precipitation cutoffs already considered by Noël et al. (2000), since this is the kind of physical situation for which the $E$-region electron heating and its effect on the conductivity gradients will have the most impact.

3.1 Case 1 - without plasma wave heating of $E$-region electrons

\subsubsection{Basic electro-dynamical response}

In Fig. 3 we present the output from TRANSCAR using only the classical electron heating rate (Eq. (8)) to describe the heating due to electric fields, five minutes after the introduction of precipitation. In Fig. 4, we present the output from ELECTRO for the same run at the same time frame. These results are consistent with those presented in our previous study (Noël et al., 2000), as well as those presented in an earlier study by St.-Maurice et al. (1996). In particular, in
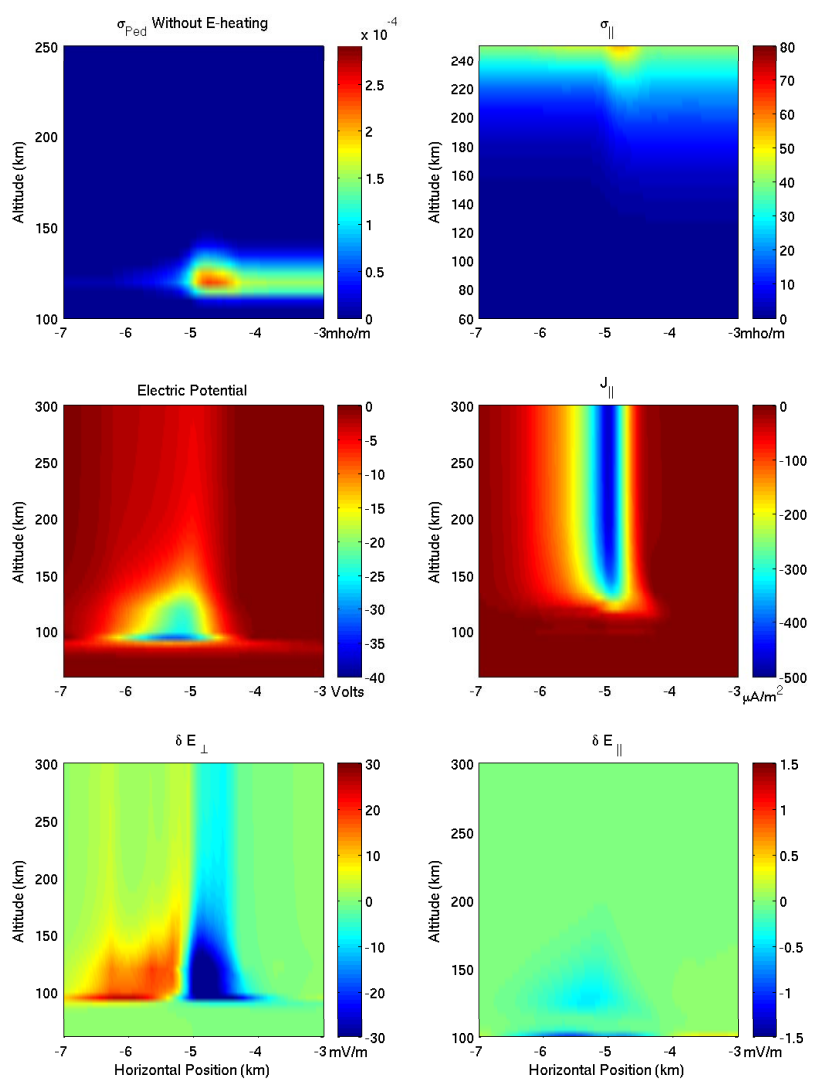

Fig. 4. ELECTRO output in the absence of $E$-region electron heating by plasma waves and with the precipitation flux given in Fig. 1.

their theoretical study, St.-Maurice et al. (1996) showed that when horizontal gradients in the conductivity existed in the presence of a large ambient electric field, field-aligned current densities of the order of a few hundred $\mu \mathrm{A} / \mathrm{m}^{2}$ would be carried by thermal electrons in the vicinity of the jump in conductivity. However, one important limitation of their model was that it was not capable of adjusting to the new currents. Consequently, the effects due to the feedbacks between the electrodynamics and the composition and energetics could not be studied with their model. Indeed, by using our model we clearly see from Fig. 4 (middle right panel) that we obtain field-aligned currents that are roughly twice as large as those from St.-Maurice et al. (1996), even though the current densities are located in the same region as those studied by St.-Maurice et al. (1996).

St.-Maurice et al. (1996) and Noël et al. (2000) were not alone in predicting large field-aligned current densities near the edges of auroral structures with sharp latitudinal precipitation cutoffs. Otto and Zhu (2001) and Zhu et al. (2001), in particular, found similar results while studying the coupling between the ionosphere and magnetosphere via Alfvén waves. The latter authors applied a shear in the plasma velocity and a horizontal magnetic field perturbation at their top boundary to launch a pair of Alfvén waves into their modelled ionosphere. These waves produced two regions of narrow field-aligned current densities centered around the 
maximum shears. After reflection of the waves from the lower $F$-region and $E$-region (their lower boundary), the field-aligned current densities intensified by $\sim 50 \%$.

\subsubsection{Electron density structures}

The two top panels of Fig. 3 provide the electron density and temperature as functions of horizontal position and altitude. The middle two panels describe the $\mathrm{O}^{+}$density and temperature while the bottom two panels show the same for $\mathrm{NO}^{+}$. In the electron density panel we clearly see the effect of the electron precipitation between 100 to $130 \mathrm{~km}$ altitude. Inside the arc, this enhancement is consistent with our choice of spectral flux in the precipitating electrons, which was shown in Fig. 1. However, near the edge of the arc, where the gradient in the precipitation is maximum, we observe a highly localized enhancement in the electron density that extends upward to $350 \mathrm{~km}$ altitude. Above $150 \mathrm{~km}$ the electron density enhancement near the edge of the arc is clearly connected to elevated electron temperatures seen in the top right panel of Fig. 3. Basically, in regions where molecular ions and their recombination control the electron density, the elevated $T_{e}$ provokes an increase in the net electron density by decreasing the molecular recombination rate (e.g., Noël et al., 2000; Sheehan and St.-Maurice, 2004). This reduction in the recombination rate can clearly be seen in the panel representing the $\mathrm{NO}^{+}$density profile (bottom left panel in Fig. 3).

Note that the enhanced electron temperatures on the edge of the arc are, in turn, due to a large and highly localized sheet of parallel current densities reaching values up to $450 \mu \mathrm{A} / \mathrm{m}^{2}$ (Fig. 4, middle right panel). The large current densities heat the electrons through friction.

There is also a secondary electron density enhancement between 250 and $350 \mathrm{~km}$ altitude around the edge of the arc. This secondary enhancement is actually related to the sharp decrease in the $\mathrm{O}^{+}$temperature seen in the middle right panel of Fig. 3. These smaller $\mathrm{O}^{+}$temperatures are directly related to a decrease in the electric field strength in that region. By generating a smaller vertical flux in the $\mathrm{O}^{+}$(i.e. a smaller ionization loss near $300 \mathrm{~km}$ ) the smaller temperatures in turn become associated with a larger density. However, below $250 \mathrm{~km}$, the conversion of $\mathrm{O}^{+}$into $\mathrm{NO}^{+}$starts to become more important than transport effects, so that the electron temperature more directly controls the net electron density through its effect on the recombination rate of molecular ions.

\subsubsection{Conductivity distribution and its impact}

The top two panels of Fig. 4 display the corresponding Pedersen and parallel conductivities. The middle two panels show the electric potential and the field-aligned current density while the bottom two panels give the associated changes that the arc has introduced in the perpendicular and parallel electric fields, respectively.

It is important in the context of the present paper to clearly understand the connection between conductivity and parallel currents. We already commented on two aspects of the direct effects on the electron density, namely:

- Below $250 \mathrm{~km}$, hot electrons, through heating from large parallel current densities, have a direct impact on the density, and therefore the conductivity, by affecting the recombination of molecular ions;

- Higher up, electric field variations introduce fluctuations in the $\mathrm{O}^{+}$temperatures which, in turn, affect their upward fluxes above $250 \mathrm{~km}$. We described how this introduced a localized density enhancement in the region between 300 and $400 \mathrm{~km}$ altitude on the inner edge of the arc.

Another factor introduces a feedback between perpendicular electric fields and the electron density, and therefore the conductivity: below $300 \mathrm{~km}$, the reaction rate that converts $\mathrm{O}^{+}$into $\mathrm{NO}^{+}$increases very rapidly, with the so-called "effective temperature" (Albritton et al., 1977; St.-Maurice and Torr, 1978; St.-Maurice and Laneville, 1998). This effective temperature is given by

$T_{e f f}=\frac{m_{n}}{m_{i}+m_{n}}\left(\frac{m_{i} U^{2}}{3 k_{b}}+T_{i}-T_{n}\right)+T_{n}$,

where $m_{n}$ and $m_{i}$ are the masses of the neutral and ion reactants, respectively, $k_{b}$ is the Boltzmann constant, $T_{i}$ and $T_{n}$ are the ion and neutral temperatures, respectively, and $U$ is the magnitude of the relative drift between the ion and neutral reactants. Note that $T_{e f f}$ should not be confused with the actual ion temperature. The effective temperature is the thermal energy of the system in the ion-neutral centre-of-mass reference frame (see St.-Maurice and Torr (1978)). For instance, in the highly collisional region below $300 \mathrm{~km}$ altitude, the ion temperature is given, to a very good degree of approximation, by the expression (St.-Maurice and Hanson, 1982)

$T_{i} \approx T_{n}+\frac{<m_{n}>}{3 k_{b}} U^{2}$,

where $<m_{n}>$ is a collision frequency-weighted average neutral mass and the relatively weak ion-electron energy exchange term has been neglected. Below roughly $300 \mathrm{~km}$ altitude, the chemical conversion of $\mathrm{O}^{+}$into $\mathrm{NO}^{+}$leads to an increase in $\mathrm{NO}^{+}$density on the inner edge of the arc.

The consequence of the temperature feedback on the conductivities is central to the present paper because it introduces a "positive feedback" into the whole system, namely, the electron density enhancements that we just described increase the Pedersen conductivity in the enhancement region (e.g. Fig. 4, top left panel). The Pedersen conductivity gradient consequently increases in the immediate vicinity, which introduces larger field-aligned current densities on the edge of the structure. In the $E$ and lower $F$-regions, the larger field-aligned currents reduce the molecular recombination through the electron temperature effect on chemistry while the associated further reductions in perpendicular electric fields on the inner edge of the arc also contribute to a further 

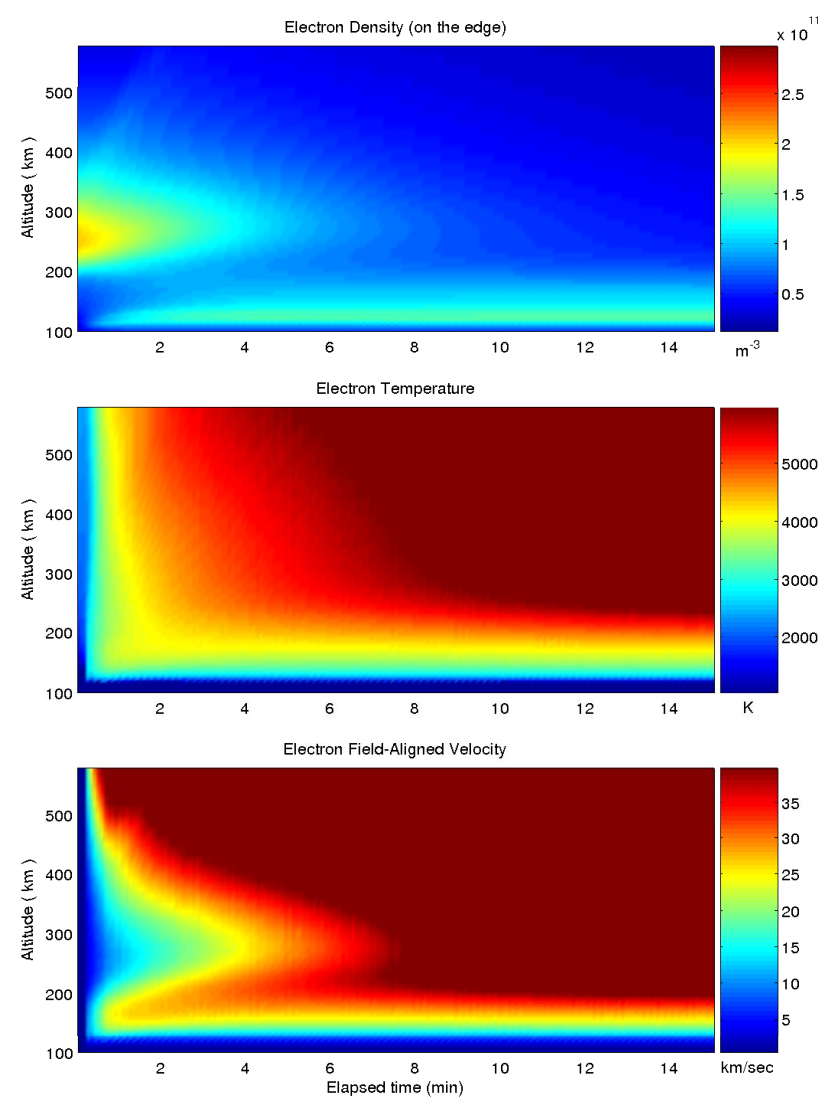

Fig. 5. Time evolution of electron parameters just on the edge of the arc, where the parallel current densities reach their peak values.

increase in the electron density in the $F$-region. Therefore, the Pedersen conductivity gradient increases further. The system becomes "unstable" in that sense, since the enhanced gradients introduce stronger parallel currents, more electron heating and so on and so forth. However, since our code is, in effect, nonlinear, the growth process reaches a limit after the structures have gone to a large enough amplitude.

For added context and for a better understanding of the processes involved we have introduced a description of the time evolution in Figs. 5 and 6 for two specific locations. In Fig. 5 we present the evolution of the electron density, temperature, and field-aligned velocity, on the edge of the arc, where the parallel currents are most intense. The changes keep pace with the increase in the conductivity gradient on the edge of the arc. Since the chemical time constants are of the order of $1 \mathrm{~min}$, it is little surprise that the parallel currents and the disturbances they introduce saturate after a few minutes. We can also clearly see from the figure that, on the edge of the arc, where the current densities reach their largest values, the minimum in the electron field-aligned velocity is located near the $F$-region peak that was present prior to the introduction of the arc.

Below $300 \mathrm{~km}$, as the $\mathrm{O}^{+}$ions are being converted into $\mathrm{NO}^{+}$ions, a secondary peak occurs in the $\mathrm{NO}^{+}$density near $200 \mathrm{~km}$ altitude (see Fig. 6). However, after the conversion of
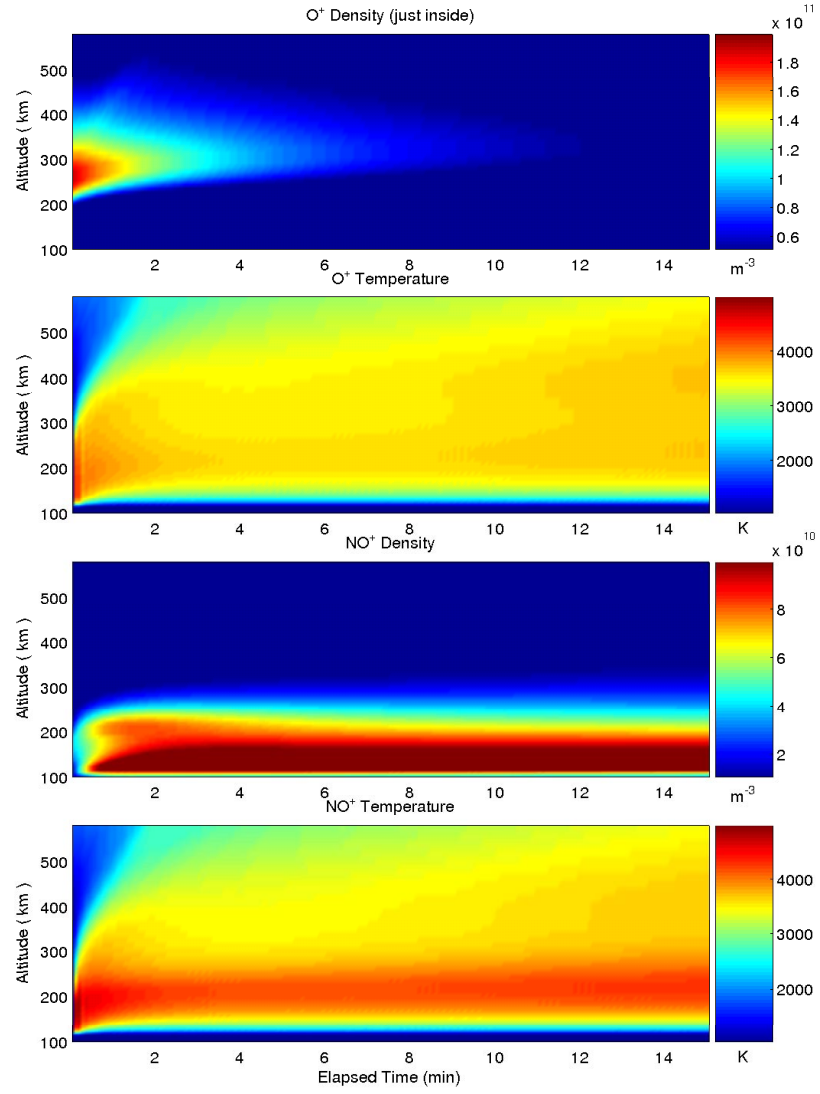

Fig. 6. Time evolution of ion parameters just inside the precipitation zone, where $E$-region conductivities reach their largest values.

$\mathrm{O}^{+}$ions into $\mathrm{NO}^{+}$ions, the initial peak disappears because of recombination. The electron velocity minimum follows suit and goes away with the disappearance of the density peak.

The conversion of $\mathrm{O}^{+}$into $\mathrm{NO}^{+}$and the resulting depletion in $F$-region densities is made particularly evident in Fig. 6, where we are showing the ion densities and temperatures just inside the arc (this location was chosen so as to clearly illustrate the changes in the ion densities in response to precipitation in addition to the rest). Figure 6 also illustrates how the ion temperature responds to the introduction of the ambient electric field and its subsequent modification after $t=0$. There is a delay in the response as the altitude increases, in accord with the fact that ion-neutral collisions totally control the response time in the initial stages of the introduction of an electric field (over much longer time scales a thermal wave is launched, much along the lines presented by Loranc and St.-Maurice (1994)). We also notice that after a short-lived initial spike the ion temperatures gradually build up in the location shown. This change is in response to a gradual increase in the perpendicular electric field with time at the location shown, just inside the arc. Finally, one might notice a 10 to $20 \%$ difference between the $\mathrm{O}^{+}$ and $\mathrm{NO}^{+}$temperatures. This difference is actually consistent with Eq. (18), being related to the weighted mass average found in that equation. It comes from the fact that the 

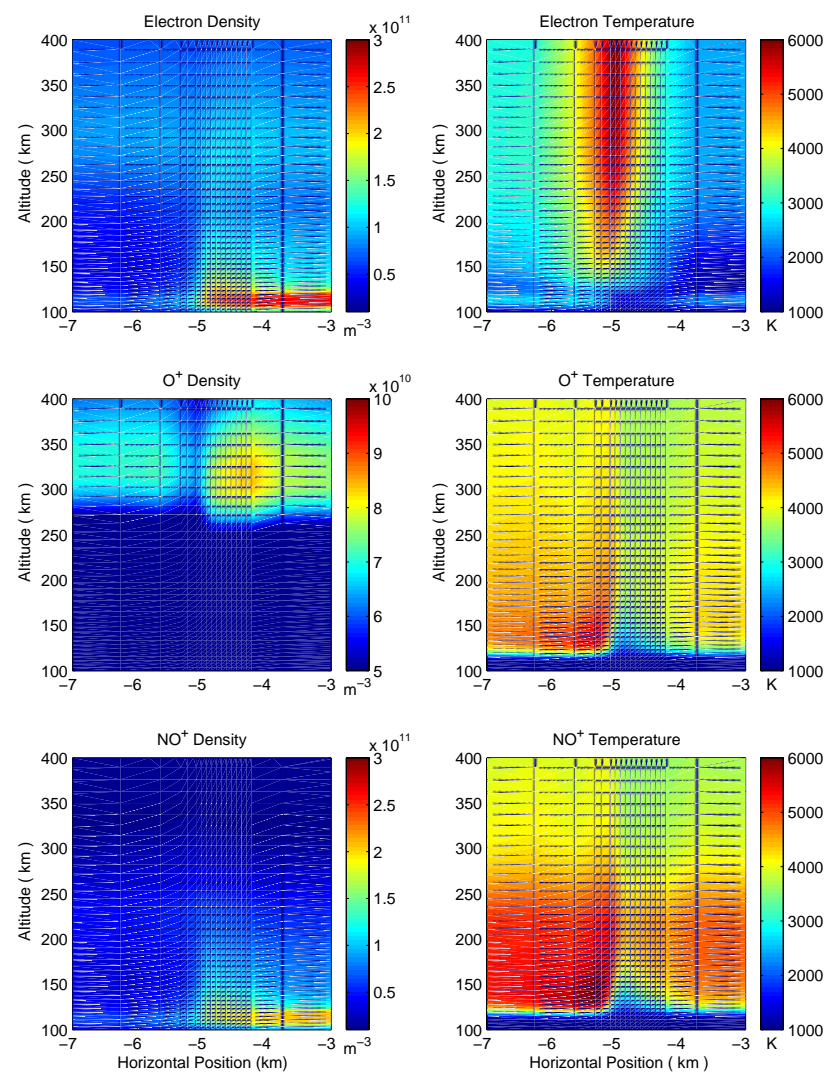

Fig. 7. Same as in Fig. 3, but with the addition of $E$-region wave heating of electrons, following the prescription proposed by Robinson (1986).

collision frequency between $\mathrm{O}^{+}$and neutral atomic oxygen is relatively large and introduces a measurable difference between the neutral mass-weighted averages of the atomic and molecular ions.

\subsection{Case 2 - with electron wave heating using Robinson's expression}

We now present in Figs. 7 and 8 the results of our calculations in the presence of wave heating rates. For our first case, we have introduced Robinson's expressions, Eq. (9), in TRANSCAR. The new figures obtained from these calculations are identical in format to Figs. 3 and 4, and the cases are identical, except for the introduction of the wave heating rate.

\subsubsection{Density differences}

When comparing the new results with the old, to start with we observe significant differences in the electron densities. Specifically, the $E$-region electron density near $120 \mathrm{~km}$ altitude nearly doubles in the precipitation region when wave heating is added (Fig. 7, top left panel) instead of being highly enhanced only near the edge of the arc in the previous case (Fig. 3, top left panel). The reason for the change
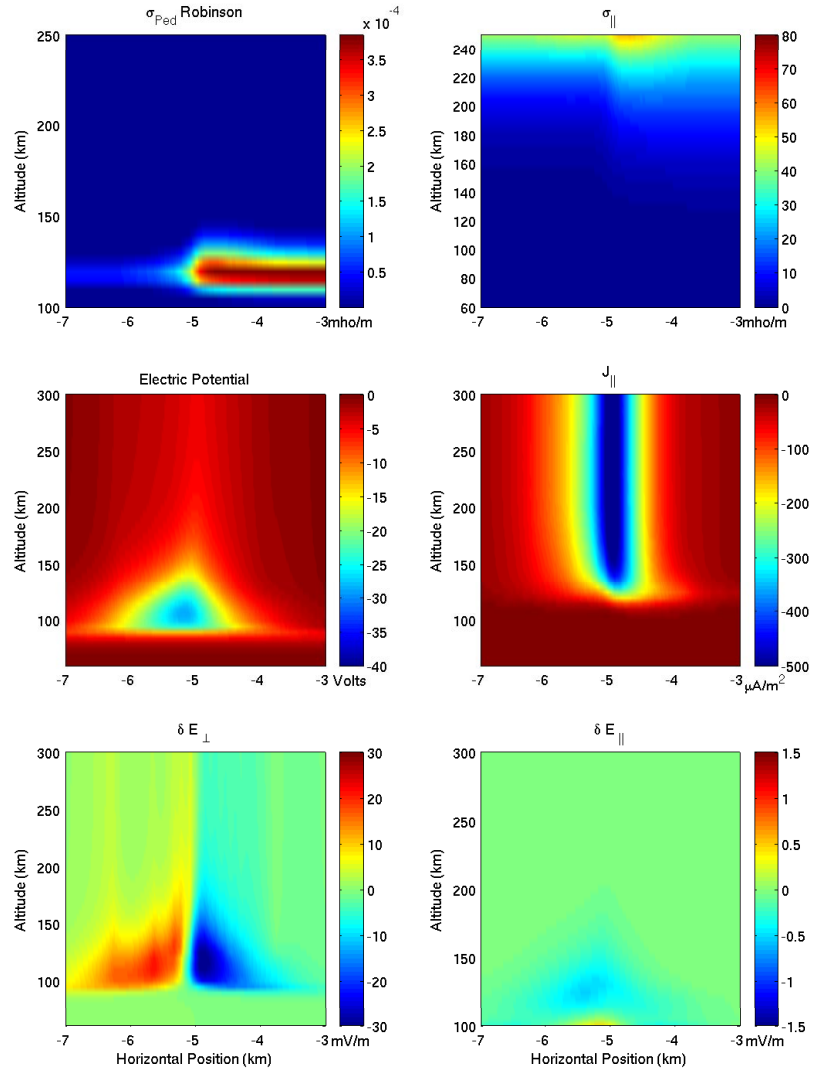

Fig. 8. Same as in Fig. 4, but with the addition of $E$-region wave heating of electrons, following the prescription proposed by Robinson (1986).

is fairly simple: with a production from precipitation, a decrease in the dissociative recombination of the molecular ions has to lead to larger densities. By contrast, outside the arc, while the recombination rate also slows down, there is no source term and the effect on the densities is considerably reduced, in spite of the elevated electron temperatures.

We also observe an increase in the electron density at about $300 \mathrm{~km}$ altitude in response to the $\mathrm{O}^{+}$density enhancement in the region of reduced electric fields. We note that the $F$-region enhancement is not as dramatic in the wave heated case as it was in the case without the wave heating source and that the wave heated case also extends a bit more horizontally. This is consistent with the $\mathrm{O}^{+}$temperature being lower over a wider horizontal distance for the second case compared to the first.

The electron density features are otherwise common to both runs. For instance, we have a density enhancement in both cases, extending upward from the $120 \mathrm{~km}$ altitude region on the edge of the arc.

\subsubsection{Temperature differences}

The electron temperatures also differ considerably between the two runs. While it may not jump out at us because of the color scales involved, it is important to notice first that in 
the wave heated case, the electrons have temperatures well in excess of $1000 \mathrm{~K}$ everywhere in a 10 to $20 \mathrm{~km}$ thick region below $120 \mathrm{~km}$ altitude. This, of course, is directly related to the wave heating term itself. A closer look also reveals that the $110-\mathrm{km}$ altitude electron temperature is significantly structured horizontally. For instance, just outside the arc, just below $\sim 120 \mathrm{~km}$ altitude, the electron temperature has a value of $\sim 3000 \mathrm{~K}$ while just inside the arc the temperature has dropped to a value of $\sim 2000 \mathrm{~K}$. The change in temperature occurs over a distance of about $400 \mathrm{~m}$. Given the strong dependence of the wave heating rate on the electric field, this change can easily be seen to be a direct consequence of the horizontal structuring in the perpendicular electric field. The latter is present in both runs but is allowed to affect the $E$ region electron temperatures only in the wave heated case.

What may be more striking is a large electron temperature increase on the edge of the arc everywhere above $150 \mathrm{~km}$. This increase is, in turn, directly related, through frictional heating, to an increase in the magnitude of the field-aligned current densities. The current density now reaches a magnitude of $\sim 550 \mu \mathrm{A} / \mathrm{m}^{2}$ (Fig. 8, middle right panel). This represents an increase of the order of $20-30 \%$ compared to the case when electron wave heating was not considered (Fig. 4, middle right panel).

In the middle and bottom right panels of Fig. 7, we observe that the $\mathrm{O}^{+}$and $\mathrm{NO}^{+}$temperatures are also more elevated than they were in Fig. 3. The temperatures are horizontally structured, as before, but they are enhanced over the case devoid of wave heating everywhere throughout the altitude range $120 \mathrm{~km}$ to $300 \mathrm{~km}$ in the horizontal interval being shown. The enhancements are particularly noticeable on the outside edge of the arc, at about $130 \mathrm{~km}$, where the temperature reaches $\sim 5500 \mathrm{~K}$ for $\mathrm{O}^{+}$and $\sim 6000 \mathrm{~K}$ for $\mathrm{NO}^{+}$. By contrast, just inside the arc the temperatures decrease to $\sim 3800 \mathrm{~K}$ for $\mathrm{O}^{+}$and $\sim 4500 \mathrm{~K}$ for $\mathrm{NO}^{+}$, over a horizontal distance of approximately $400 \mathrm{~m}$, respectively. The temperature enhancements are due to changes introduced in the perturbed electric field in the wave heated case. Basically, the regions of positive electric field enhancements are more pronounced than in the wave-heating-free case, whereas the regions of negative enhancements are less depressed than before.

\subsubsection{Electrodynamical changes}

The electric field differences that we have just mentioned are, of course, associated with visible changes in the potential, as can be seen from a comparison of the middle left panels of Fig. 4 and Fig. 8. Most noticeable is a motion upward of the potential maximum in the wave heated case. The maximum is now near $110 \mathrm{~km}$, namely around the point where the electron temperature is also peaking. This affects the vertical distribution in the parallel field, in particular. The effect is most visible where it concerns the parallel currents, with the $20-30 \%$ increase in current density that we already mentioned for the wave heated case.
The changes in the electric field are connected, in turn, to changes in the conductivity gradient, as can be readily seen from Eq. (6). Figure 8 clearly shows that the Pedersen conductivity in the wave heating case is nearly 5 times higher inside the arc than outside. The enhancement in the Pedersen conductivity inside the arc results in larger horizontal gradients on the edge of the arc. The large gradients in the conductivity is at the origin of the parallel currents' intensification. We also notice that, contrary to the case without wave heating, the Pedersen conductivity is also fairly constant throughout the arc. The increase in the conductivity is, in large part, directly related to the large increase in the $E$ region electron density. There is, nevertheless, also a more minor contribution from the elevated electron temperature, as can be seen from Eqs. (14) to (16).

The enhancements in the $E$-region conductivities inside the arc have consequently to move more charges to the edge of the arc. A priori, this can have two different kinds of consequences concerning the electrodynamics. First, one could argue that with higher Pedersen conductivities, the ambient (perpendicular) electric field inside the arc should be shorted out more than in the poorer conductivity case. Alternatively, the effect could be reduced if, instead, the difference translated into more intense parallel fields and parallel currents on the edge of the arc. Clearly, at least for the case that we have considered here, the second process dominates: The perpendicular electric field panels only show a small reduction in the sense that the perpendicular field becomes reduced further inside the arc than in Case 1. However, the magnitudes of the perpendicular fields are comparable in both runs. Where parallel currents and parallel electric fields on the edge of the arc are concerned, however, we have a different story. The parallel current densities are measurably larger in the wave heating case. This is, of course, due to the presence of stronger parallel electric fields which may be less easy to see, but are, nevertheless, very much there.

We conclude that there can be little doubt that $E$-region plasma wave heating does affect the response of the plasma to sharp changes in precipitating fluxes in the presence of strong ambient electric fields. While quite visible effects are seen in various locations in the densities and temperatures, the electrodynamics at the edge of the arcs is most strongly affected. Our study of the results furthermore leads us to conclude that the enhanced parallel currents and fields are mostly the consequence of an enhancement in the horizontal (perpendicular) Pedersen conductivity gradient at the edge of the arc.

As with the run made without wave heating, we have also monitored, for added perspective, the time evolution of the system on the edge of the arc, near the region of maximum parallel current densities, and just inside the arc, in the region of largest Pedersen conductivity enhancements. These results are shown in Figs. 9 and 10 and can be compared with what we presented in Figs. 5 and 6. Clearly, the case with wave heating evolves more quickly on the edge of the arc: with the stronger perpendicular fields the conversion of $\mathrm{O}^{+}$ into $\mathrm{NO}^{+}$is faster and the $F$-region peak disappears more 

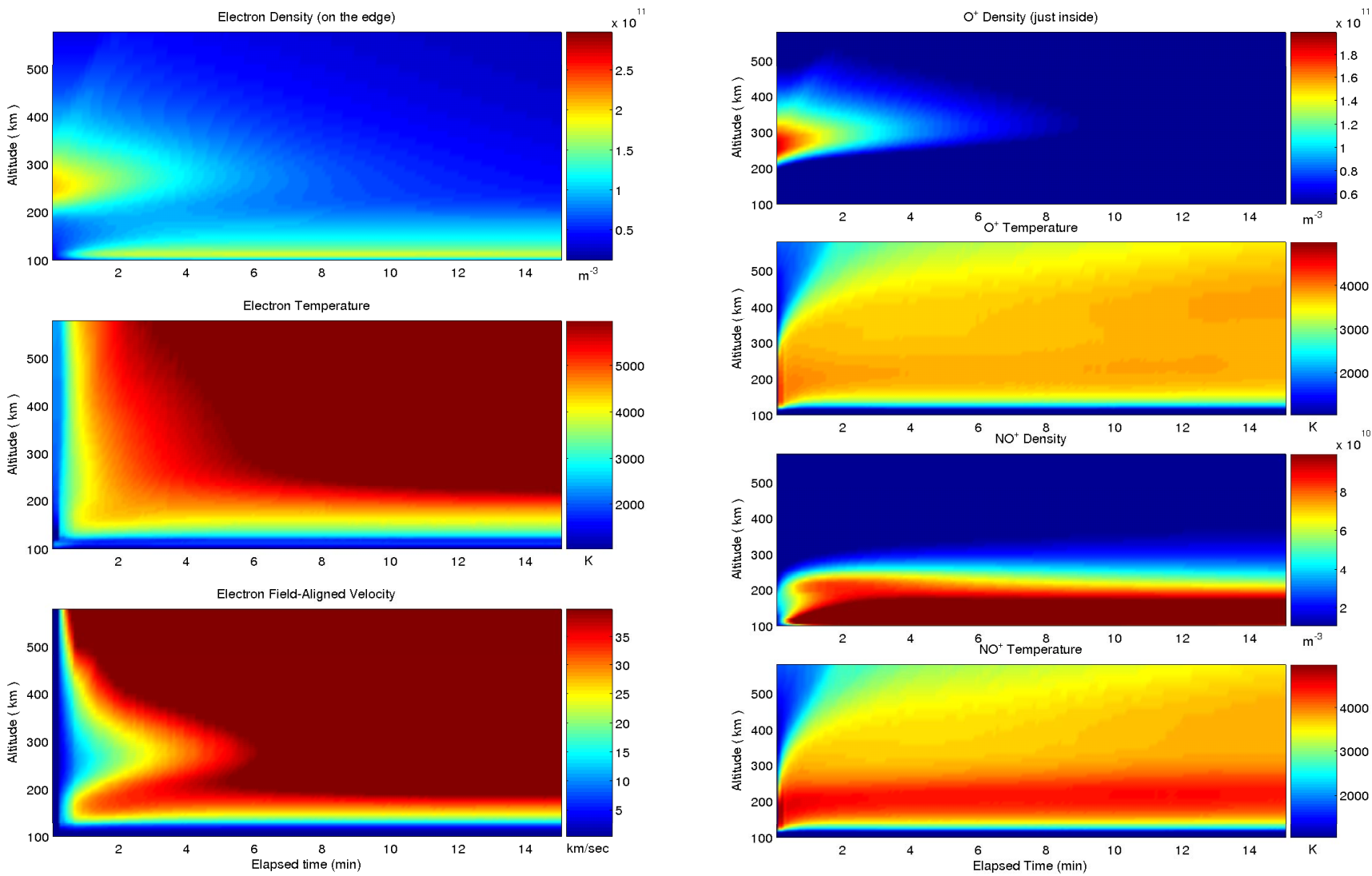

Fig. 9. Time evolution of electron parameters just on the edge of the arc, where the parallel current densities reach their peak values.

quickly. We can also see that the changes in the $\mathrm{NO}^{+}$density inside the arc are evident right from the beginning, since $E$-region electron heating responds immediately to applied perpendicular fields and therefore immediately slows down the $\mathrm{NO}^{+}$recombination process, thereby allowing more ions to be present.

3.3 Case 3 - with electron wave heating using the Dimant and Milikh's expression

As stated in the Introduction, the expressions for the wave heating rate that were obtained by Robinson (1986) are different from those obtained by Dimant and Milikh (2003). Since both formulations appear to do a reasonable job where observations are concerned, we have decided to repeat our wave heating run with the Dimant and Milikh rates, to see if whatever differences there are can have an impact on the response of the system.

It turns out that the results from the two heated runs, while not identical, are rather similar. The largest differences are in the electrodynamical part, but even there, they are small. For instance, the field-aligned current density was $\sim 550 \mu \mathrm{A} / \mathrm{m}^{2}$ when we used Robinson's expressions, while for the Milikh and Dimant (2003) case the magnitude reaches $\sim 570 \mu \mathrm{A} / \mathrm{m}^{2}$. This is still only a $\sim 4 \%$ increase. The small

Fig. 10. Time evolution of ion parameters just inside the precipitation zone, where $E$-region conductivities reach their largest values.

differences can themselves be tracked down to small changes in the conductivity distribution.

In order to more clearly assess the differences between our two wave heating cases, we have plotted in Fig. 11 the differences in the electrodynamical parameters between the two runs. The Pedersen and parallel conductivities differences are shown in the top panels. The middle panels contain the electric potential (left side) and the absolute value of the differences in the field-aligned current density (right side). The bottom panel contain the differences in the perturbed perpendicular and parallel electric fields. The Milikh and Dimant (2003) rates produce somewhat larger temperatures than the Robinson model. This, in turn, give somewhat larger Pedersen conductivities in the former case, which results in somewhat larger parallel electric fields and somewhat less contrast in the perpendicular electric fields on each side of the edge of the arc. These trends are similar to the differences that were observed between the heated and non-heated case, but are, of course, of much smaller amplitudes.

Finally, as a way to summarize the key features introduced by wave heating, and in order to make the comparison more precise, we have introduced profiles of the main ion and electron parameters at the edge of the arc and just inside the arc at the 5-min mark used for the color plots. We have compared our three runs, namely, the one without heating and the 

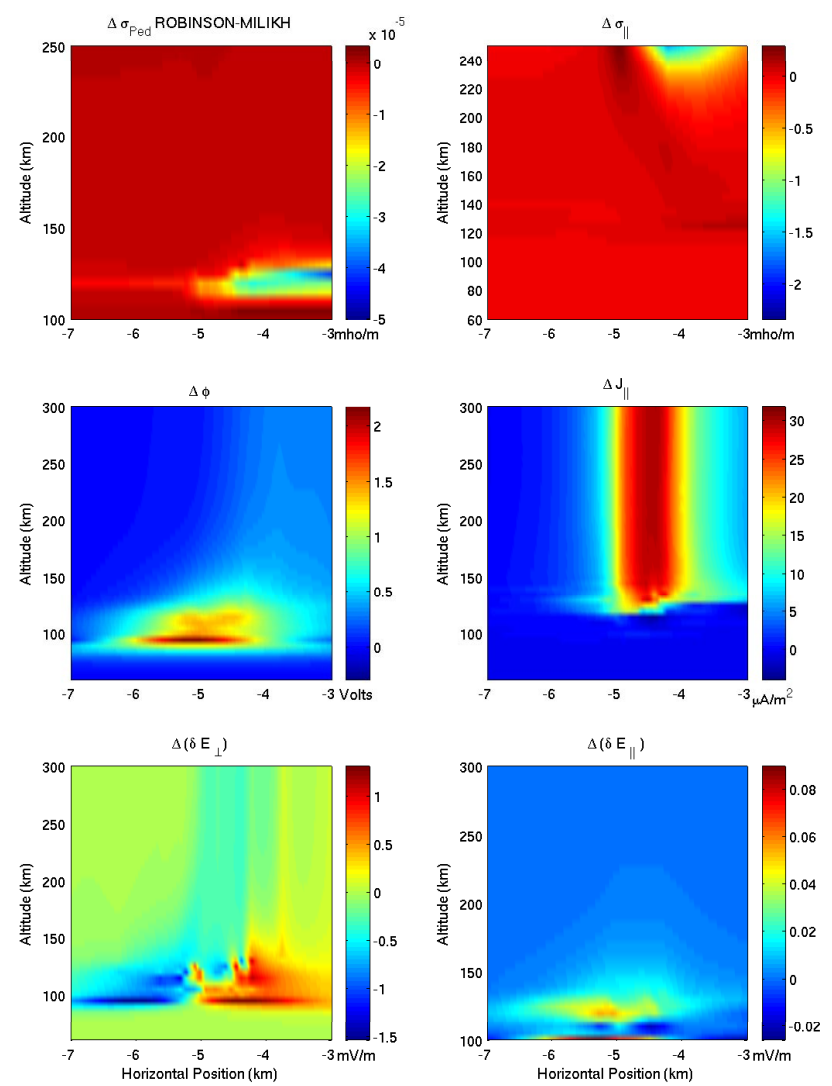

Fig. 11. Differences between various electrodynamical parameters for the two different wave heating rate expressions (case 2 results minus case 3 results) that we have considered in this work. Same presentation format as in Fig. 4 but with the differences instead of the actual quantities.

other two runs into which the wave heating were parameterized somewhat differently. The results are shown in Figs. 12 and 13. They illustrate more precisely the small differences between the two heating runs (some curves are exactly on top of each other) while showing the effect of $E$-region wave heating on the results in these two key locations.

\section{Summary and conclusion}

We have used our two-dimensional model of small-scale electrodynamics near auroral arcs to assess the impact of electron heating by plasma waves in the auroral $E$-region. Just as we did in the Noël et al. (2000) study, we focused on arcs with sharp cutoffs in precipitation with little structure inside the precipitation, region, since these are the kinds of arcs that are associated with strong parallel current densities on their edges and are very sensitive to Pedersen conductivity gradients.

In the present work, electron temperatures that are boosted by $E$-region wave heating will, in turn, boost the electron density inside the precipitating regions by decreasing the recombination rate of the molecular ions. This increases the
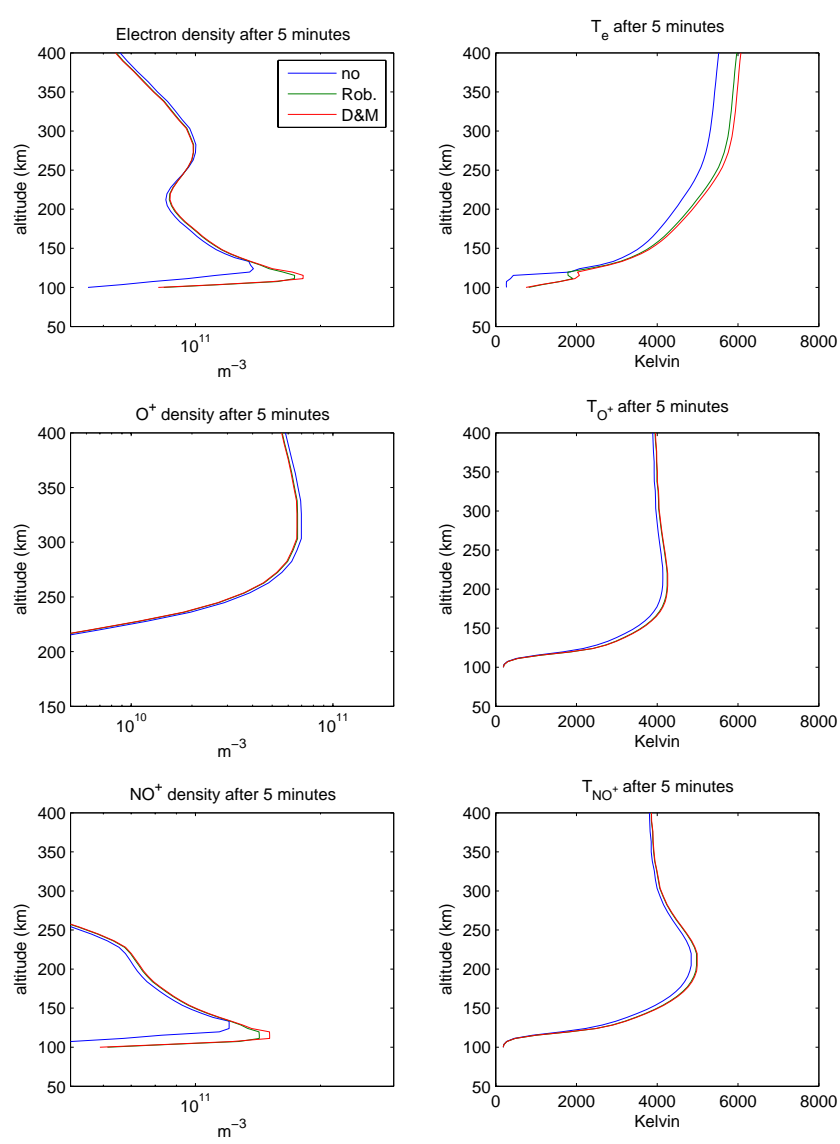

Fig. 12. Density and temperature profiles 5 min after the introduction of an electric field and of precipitation, taken just on the edge of the arc, in the region of large parallel current densities.

contrast between the Pedersen conductivity inside the arc versus outside the arc. In turn, this drives a horizontal divergence in perpendicular currents near the edge of the arcs, which ends up triggering stronger parallel current densities in that region. Additional feedback effects are introduced because the parallel currents create, in turn, a heating of electrons through friction, thereby affecting collision frequencies and densities, which in turn again affects the conductivity of the medium. With our imposed 200-m cutoff scale for precipitation, the resulting effects are very large, increasing already substantial parallel current densities of several hundred $\mu \mathrm{A} \mathrm{m}^{-2}$ by up to $30 \%$.

We used two different expressions for the wave heating rate, based on two physically very different models that have been used to describe the process. Our results are essentially the same for the two models. This should not be too surprising, since both sets of formulae appear to fit the observations reasonably well, meaning that the results are essentially the same, in spite of a different physics. This is, nevertheless, an important point, particularly if we favor the second mechanism, where the waves are unable to substantially modify the conductivities directly through anomalous transport properties. Our model calculations illustrate that the large 

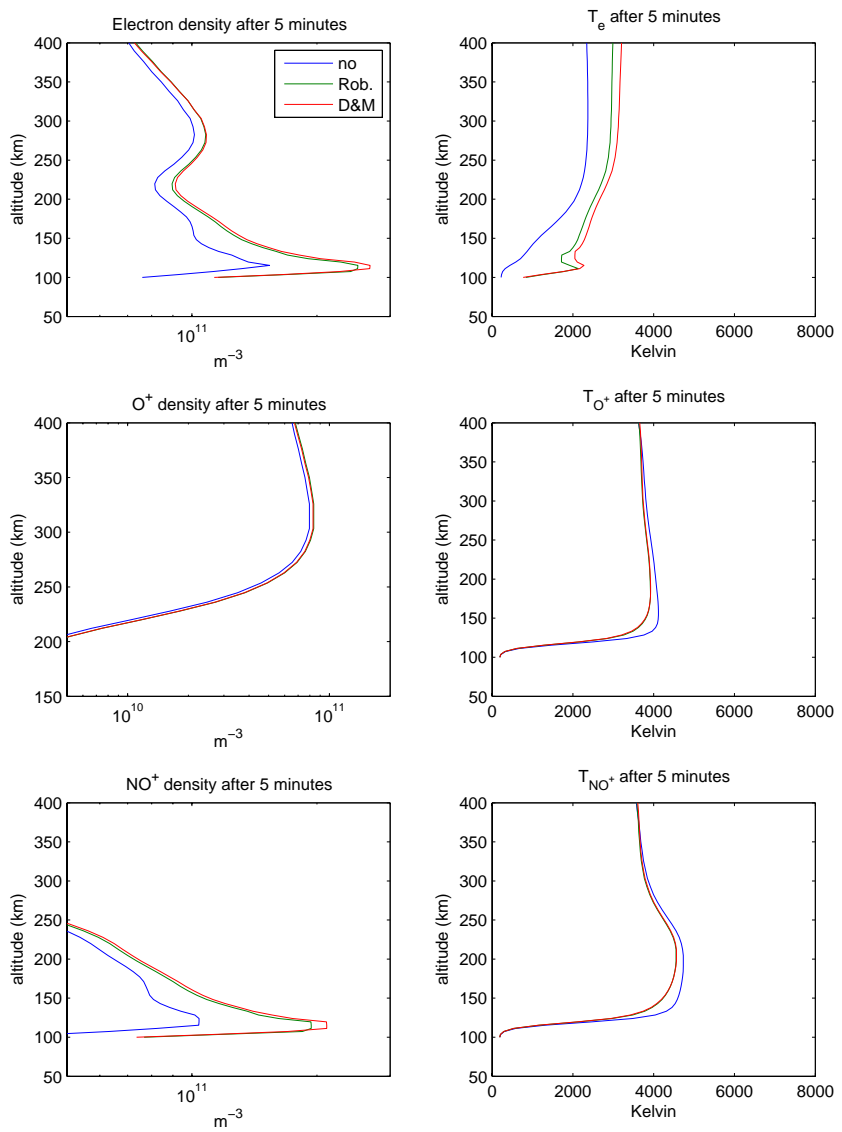

Fig. 13. Density and temperature profiles 5 min after the introduction of an electric field and of precipitation, taken just inside the arc, in the region of peak $E$-region electron densities.

amplitude, small-scale plasma structures can play an important role not so much by directly affecting the electric field that creates them, but more by modifying the fields through changes in the conductivities, with conductivity gradients introduced indirectly through chemical effects.

Acknowledgements. This research was funded by research grants to JMN and JPSTM from the National Science and Research Engineering Council of Canada.

Topical Editor M. Lester thanks Y. Dimant and another referee for their help in evaluating this paper.

\section{References}

Albritton, D. L., Dotan, I., Lindinger, W., McFarland, M., Tellinghuisen, J., and Fehsenfeld, F. C.: Effects of ion speed distributions in flow-drift tube studies on ion-neutral reactions, J. Chem. Phys., 66, 410-421, 1977.

Blelly, P.-L. and Schunk, R. W.: A comparative study of the timedependent standard 8-, 13- and 16-moment transport formulations of the polar wind, Ann. Geophys., 11, 443-469, 1993.

Blelly, P.-L., Robineau, A., Lilensten, J., and Lummerzheim, D.: 8-moment fluid models of the terrestrial high latitude ionosphere between 100 and $3000 \mathrm{~km}$, Solar terrestrial energy program (STEP): handbook of ionospheric models, 53-72, 1996.
Blelly, P.-L., Lilensten, J., Robineau, A., Fontanari, J., and Alcaydé, D.: Calibration of a numerical ionospheric model with EISCAT observations, Ann. Geophys., 14, 1375-1390, 1996, SRef-ID: 1432-0576/ag/1996-14-1375.

Blelly, P.-L., Lathuillère, C., Emery, B., Lilensten, J., Fontanari, J., and Alcaydé, D.: An extended TRANSCAR model including ionospheric convection: simulation of EISCAT observations using inputs from AMIE, Ann. Geophys., 23, 419-431, 2005, SRef-ID: 1432-0576/ag/2005-23-419.

Dimant, Y. S. and Milikh, G. M.: Model of anomalous electron heating in the $E$ region, I: basic theory, J. Geophys. Res., 108, 1350, doi:10.1029/2002JA009,524, 2003.

Lilensten, J., Kofman, W., Wisenberg, J., Oran, E., and Devore, C.: Ionization efficiency due to primary and secondary photoelectrons: a numerical model, Ann. Geophys., 7, 83-90, 1989.

Lilensten, J. and Blelly, P. L.: The TEC and F2 parameters as tracers of the ionosphere and thermosphere, J. of Atmos. Terr. Phys., 64, 775-793, 2002.

Loranc, M. and St.-Maurice, J.-P.: A time-dependent gyro-kinetic model of thermal ion upflows in the high-latitude $F$-region, J. Geophys. Res., 99, 17 429-17451, 1994.

Lummerzheim, D. and Lilensten, J.: Electron transport and energy degradation in the ionosphere: evaluation of the numerical solution, comparison with laboratory experiments and auroral observations, Ann. Geophys., 12, 1039-1051, 1994,

SRef-ID: 1432-0576/ag/1994-12-1039.

Milikh, G. M. and Dimant, Y. S.: Kinetic model of electron heating by turbulent electric field in the $E$ region, Geophys. Res. Lett., 29, doi:10.1029/2001GL013,935, 2002.

Milikh, G. M. and Dimant, Y. S.: Model of anomalous electron heating in the $E$ region, II: detailed numerical modeling., J. Geophys. Res., 108, 1351, doi:10.1029/2002JA009,527, 2003.

Noël, J.-M., St.-Maurice, J.-P., and Blelly, P.-L.: Nonlinear model of short-scale electrodynamics in the auroral ionosphere, Ann. Geophys., 18, 1128-1144, 2000,

SRef-ID: 1432-0576/ag/2000-18-1128

Otto, A. and Zhu, H.: Fluid plasma simulation of coupled systems: Ionosphere and magnetosphere, in: Space Plasma Simulation, Proc. International School for Space Simulation, (Eds.) Buechner, J., Dunn, G. T., and Scholer, M., 6th International School/Symposium on Space Plasma Simulation, Copernicus Gessellschaft, Germany, 96, 2001.

Rees, M. H.: Physics and chemistry of the upper atmosphere, Cambridge atmospheric and space science series, Cambridge University Press, Cambridge, United Kingdom, 1989.

Robinson, T. R.: Towards a self-consistent nonlinear theory of radar auroral backscatter, J. Atmos. Terr. Phys., 48, 417-423, 1986.

Schlegel, K. and St.-Maurice, J.-P.: Anomalous heating of the polar $E$-region by unstable plasma waves, 1 . Observation, J. Geophys. Res., 86, 1447-1452, 1981.

Schunk, R. W. and Nagy, A. F.: Ionospheres. Physics, plasma physics, and chemistry, Cambridge atmophseric and space science series, Cambridge University Press, Cambridge, United Kingdom, 2000.

Sheehan, C. and St.-Maurice, J.-P.: The dissociative recombination of $\mathrm{N}_{2}^{+}, \mathrm{O}_{2}^{+}$, and $\mathrm{NO}^{+}$: Rate coefficients for ground state and vibrationally excited ions, J. Geophys. Res., A03302 doi:10.1029/2003JA010132, 2004.

St.-Maurice, J.-P.: A unified theory of anomalous resistivity and Joule heating effects in the presence of $E$-region irregularities, J. Geophys. Res., 92, 4533-4542, 1987.

St.-Maurice, J.-P.: Wave-induced diffusion in the turbulent $E$ - 
region, in: Polar cap Dynamics and High Latitudes turbulence: SPI Conference proceedings and Reprint series, No. 8, 1988, Scientific Publishers, Cambridge, Mass., 323-348, 1990a.

St.-Maurice, J.-P.: Electron heating by plasma waves in the high latitude $E$-region: Theory, in: Advances in Space Research, vol. 10, Pergamon Press 239-249, 1990 b.

St.-Maurice, J.-P. and Hamza, A. M.: A new nonlinear approach to the theory of $E$ region irregularities, J. Geophys. Res., 106, 1751-1759, 2001.

St.-Maurice, J.-P. and Hanson, W. B.: Ion frictional heating at high latitudes and its possible use for an in situ detemination of neutral thermospheric winds and temperatures, J. Geophys. Res., 87, 7580-7602, 1982.

St.-Maurice, J.-P. and Laher, R.: Are observed broadband plasma wave amplitudes large enough to explain the enhanced electron temperatures of the high-latitude $E$ region?, J. Geophys. Res., 90, 2843-2850, 1985.
St.-Maurice, J.-P. and Laneville, P. J.: The reaction rate of $\mathrm{O}^{+}$with $\mathrm{O}_{2}, \mathrm{~N}_{2}$, and NO under highly disturbed auroral conditions., J. Geophys. Res., 103, 17 519-17 521, 1998.

St.-Maurice, J.-P. and Torr, D. G.: The effect of relative speed distributions on the reaction rates of $\mathrm{O}^{+}$with $\mathrm{N}_{2}, \mathrm{O}_{2}$ and $\mathrm{NO}$ in the thermosphere, J. Geophys. Res., 83, 969-977, 1978.

St.-Maurice, J.-P., Kofman, W., and James, D.: In-situ generation of intense parallel fields in the lower ionosphere, J. Geophys. Res., 101, 335-356, 1996.

Wickwar, V. B., Lathuillère, C., Kofman, W., and Lejeune, G.: Elevated electron temperatures in the auroral $E$ layer measured with the chatanika radar, J. Geophys. Res., 86, 4721-4730, 1981.

Zhu, H., Otto, A., Lummerzheim, D., Rees, M. H., and Lanchester, G.: Ionosphere-magnetosphere simulation of small-scale structure and dynamics, J. Geophys. Res., 106, 1795-1806, 2001. 